\title{
HACIA UNA GESTIÓN EFICIENTE EN EL SECTOR PÚBLICO
}

Lucas Navarro, académico FEN UAH y Mauricio Tejada, académico FEN UAH.

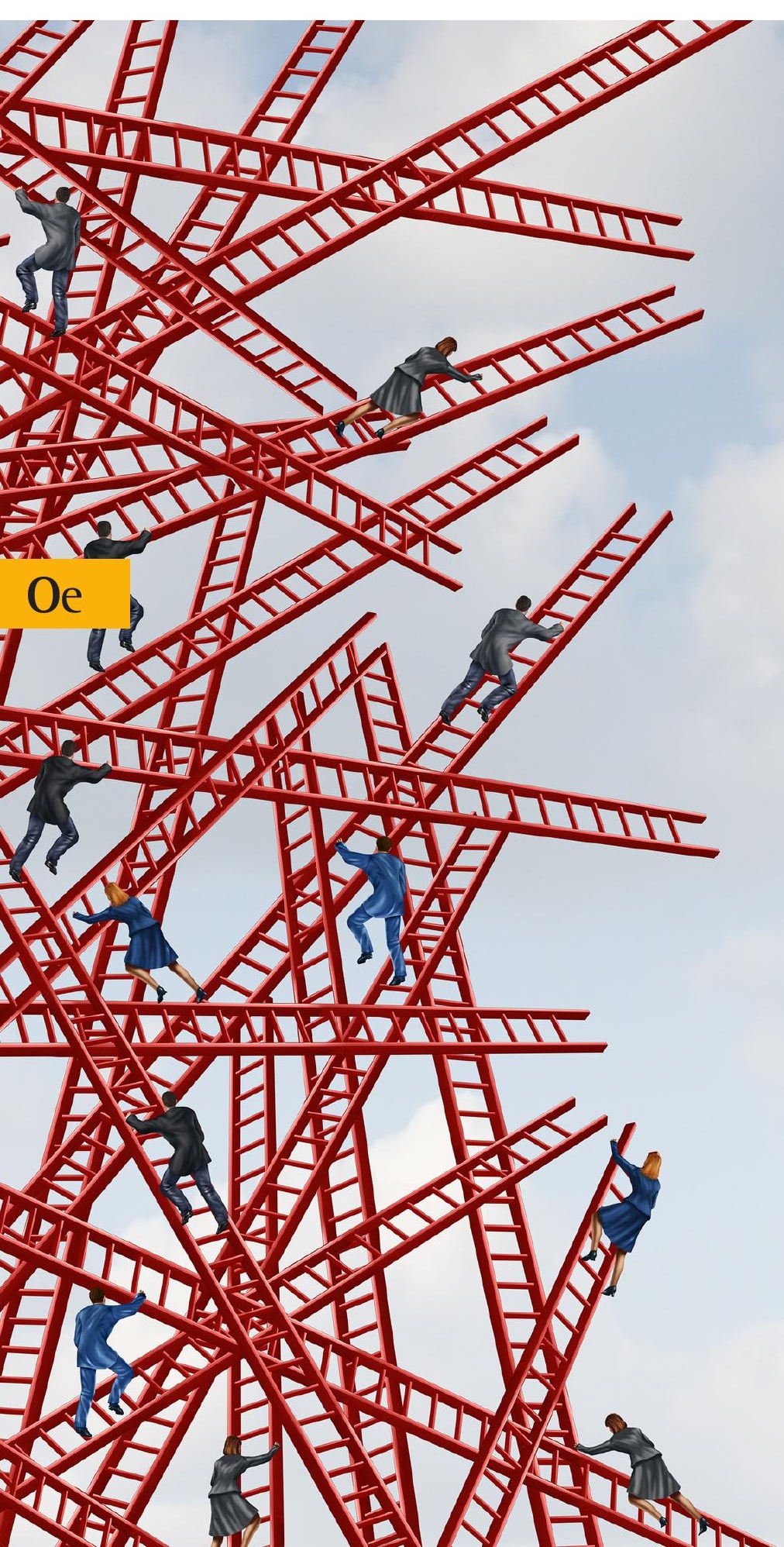

El sector público es uno de los principales empleadores del mercado laboral en el mundo. Chile no es la excepción. Considerando los últimos datos de la Nueva Encuesta Suplementaria de Ingresos disponibles para 2016 (ver Tabla 1), más de un 12\% de los hombres ocupados asalariados en edad adulta trabajan en el sector publice. Este porcentaje sube a más del 20\% si se consideran trabajadores ancidencia del empleo pur mas. Es destacable talmativamente constante en los últimos años. Por otro lado, los salarios promedio del sector público en 2016, para el mismo grupo de trabajadores considerado antes fueron $33 \%$ superiores a los del sector privado asalariado. Más aún, trabajadores sin educación superior ganaban en promedio $25 \%$ más en el sector público mientras que los trabanos en comp on educacion tercianta ganaban cerca de $8 \%$ meos diferenciales salariales en favor del sector público eran todavia más abutados en años anteriores.

En un proyecto de investigación en el que estamos trabajando estimamos la productividad del trabajo en los sectores público y privado, para asalariados hombres en edad adulta y para los dos grupos según nivel educativo considerados. Si bien los datos de las encuestas de hogares y empleo no permiten conocer la distribución de los trabajadores del sector público por tipo de contrato, ellos resultan utiles para caracterizar al empleo en este sector. La Figura 1 presenta información sobre la composición del empleo por nive educativo en ambos sectores. Los datos muestran una mejor composición del empleo por nivel educativo en el sector publico que en el privado en todos los años considerados. En efector, mientras 37\% de los trabajadores del sector pulico tienen un nivel educativo alto, en

También encontramos, al iual que en otros estudios para chile que existe un premio por trabajar en sector público (mayor los trabajadores de alto nivel educativo) y que dichos trabajos tienden a ser mucho más estables que los del sector privado. En efecto la duración promedio estimada de los trabajos en los sectores publico y privado es de 46 y 22 meses, respectivamente.

Por su parte, las estimaciones de productividad arrojan resultados llamativos. En el grupo de alta calificación, encontramos que los trabajadores en el sector privado son en promedio $37 \%$ más productivos que en el sector público. Estas diferencias de productividad en favor del sector privado son incluso mayores al considerar sólo al grupo de trabajadores de menor nivel edu cativo. Aqui surgen varias preguntas: ¿cómo se interpretan esas

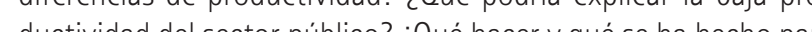

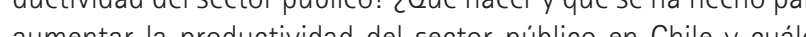
aunentar la productivida

En términos generas?

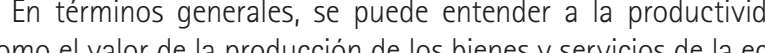
nomia, en relación a los insumos utilizados para obtenerlos. Asi productividad del trabajo representa el valor de lo que produce en promedio cada trabajador. Para poder medirla es necesario en primer lugar podervalorar la producción. Esta tarea es facil en el tor privado, en donde se producen bienes y servicios que se venden a distintos precios en los mercados. El problema es como valorar-os ienes y servicios que produce el sector público, que en su mayoria 作 en sector privado.

Por un tado, el sector público produce bienes que complementan produccion del sector privado (como en el caso de la educación v la salud en Chile) y. por el otro, el sector publico produce bienes . vato, como por ejemplo la justicia, el registro civil, las prisiones, es defersa naciona, los cuerpos diplomaticos, ete. En estos casos a que nos perata tstado, ya que no existe un mercado de justicia

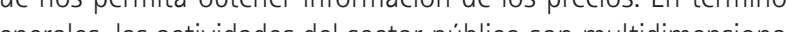
les e intrinsecamente dificiles de valorar, lo que hace compleja la ne la productividad.

te Ención, a partir de la información disponible de salarios $y$ de rgumentos teóricos sobre cómo se determinan los mismos, para estimar la productividad de los trabajadores en ambos sectores. Los resultados indican que en términos esperados, si comparamos dos

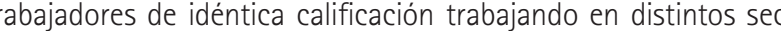
ares, el empleado en el sector privado será más productivo que -

cursos de manera eficiente si produce lo que desea con la menor cantidad de insumos posible. Se po-

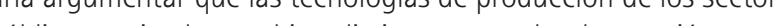
pector y privo es mon bies distinas y que la obsenvarion que el Estado sea ineficiente. Sin embargo, el hecho de no poder medir ácilmente lo que producen las instituciones públicas, y de que no . os incentivos adecuados para que el Estado produzca los bienes y la sociedad le demanda de manera eficiente.

Ante esto, desde la década de los noventa en muchos paises desarrollados se vienen implementando esquemas de incentivos en el sector público. No obstante, el contar con esquemas de pagos por incentivos adecuados en el sector público es sumamente dificil. Raramente es posible vincular los contratos de trabajo a esfuerzo y el desempeño. Tambien es dificil restringir la introistón y la arbitrariedad del poder politico en la gestion de las . abear obtivos, un desafio no menor es que las metas repreEsto es mucho más sencillo en el sector privado, donde el objetivo es maximizar ganancias. El sector público produce bienes servicios que no siempre tienen valores de referencia fácilmente identificables. En este contexto, un sistema de incentivos para una repartición pública basado en metas, puede generar efectos (a) si los indicadores considerados no reflejan adecuadamente el "valor" que genera esa agencia pública para la sociedad. Esta agencia puede terminar alterando su misión de servi la sociedad en múltiples dimensiones por el cumplimiento de metas predefinidas a partir de esos indicadores. Por ejemplo, suponga que el personal de Carabineros provee sólo los servicios cada multa por infracciones a esta ley. Esto puede ser un buen 
incentivo para aumentar los controles de ponder una solicitud diciendo que los datos te, más allá de su cumplimiento, el comtrânsito, pero al mismo tiempo un incen- no estann disponibles, como comunmente ponente variable de las remuneracion

tivo a descuidar la prevención del delito. es la experiencia de muchos que piden in- del sector público asociado directamente tro problema es que en muchas agencias la producción resulta de esfuerzos grupaes y por lo tanto los incentivos debiera (tules. Est grupales, en lugar de indivicuales. Esto hace tambièn que existan trasabiendo que sus cons a sub-esforzarse sán as metas. Anter para que los sistemas de incentivos generen os efectos deseados en la productividad de sector público. En Chile existen tres grandes mecanismos de incentivos: un incentivo individual al Alto Directivo Público, un inentivo colectivo (convenios de desempeño colectivo de $8 \%$ de la remuneración base anual) y un incentivo institucional, entre los que se destaca el Programa de Mejoramiento de la Gestion (PMG, de hasta 7,6\% como el de mayor prevalencia. Este progra ma, vigente desde fines de los noventa, es peñacter grupal y estáa asociado al desemparticipantes Las motas son definidas por pas propios equipos con el asesoramiento de técnicos especializados, y la evaluación del cumplimiento de metas es realizada por terceros. Pueden calificar al program trabajadores de planta y a contrata. Hacia 2015 el PMG alcanzaba 127 instituciones casi 100 mil empleados publicos. Los otros programas de incentivos abarcan 41 instituciones más. En total, estos sistemas se aplicaron en el 83\% de las instituciones las que se les asignaron recursos en la Ley

Resulta llamativo que el 98\% de las reparticiones alcanzaron a cumplir con las A la luz de los últimos comos anteriores. preguntarse en qué medida las metas que se definieron representan de manera adecuada la productividad con la que se dese que el sector público gestione los recursos que la sociedad le transfiere con sus impuestos. A modo de ejemplo, el Sistema de Acceso a la Información Pública utiliza como indicador de calidad de atención a los usuarios el "\% de solicitudes de acceso a la informacion publica respondidos en hasta formacion por transparencia, se puede ha- al desempeño individual en gestión sigue er en mucho menos de 15 dias habiles y siendo bajo. En cambio, existe una gran cantidad de asignaciones que se suman a sueldo base pero que no necesariamente En lineas generales, las metas del PMG tienen que ver con la eficiencia en la gespalo pare tacles de cumplir y reflejan tion. En definitiva, queda mucho por hacer instituciones participantes. Adicionalmen- vos en el sector público.

Tabla 1: Diferencial Salarial e Incidencia del Empleo Püblico
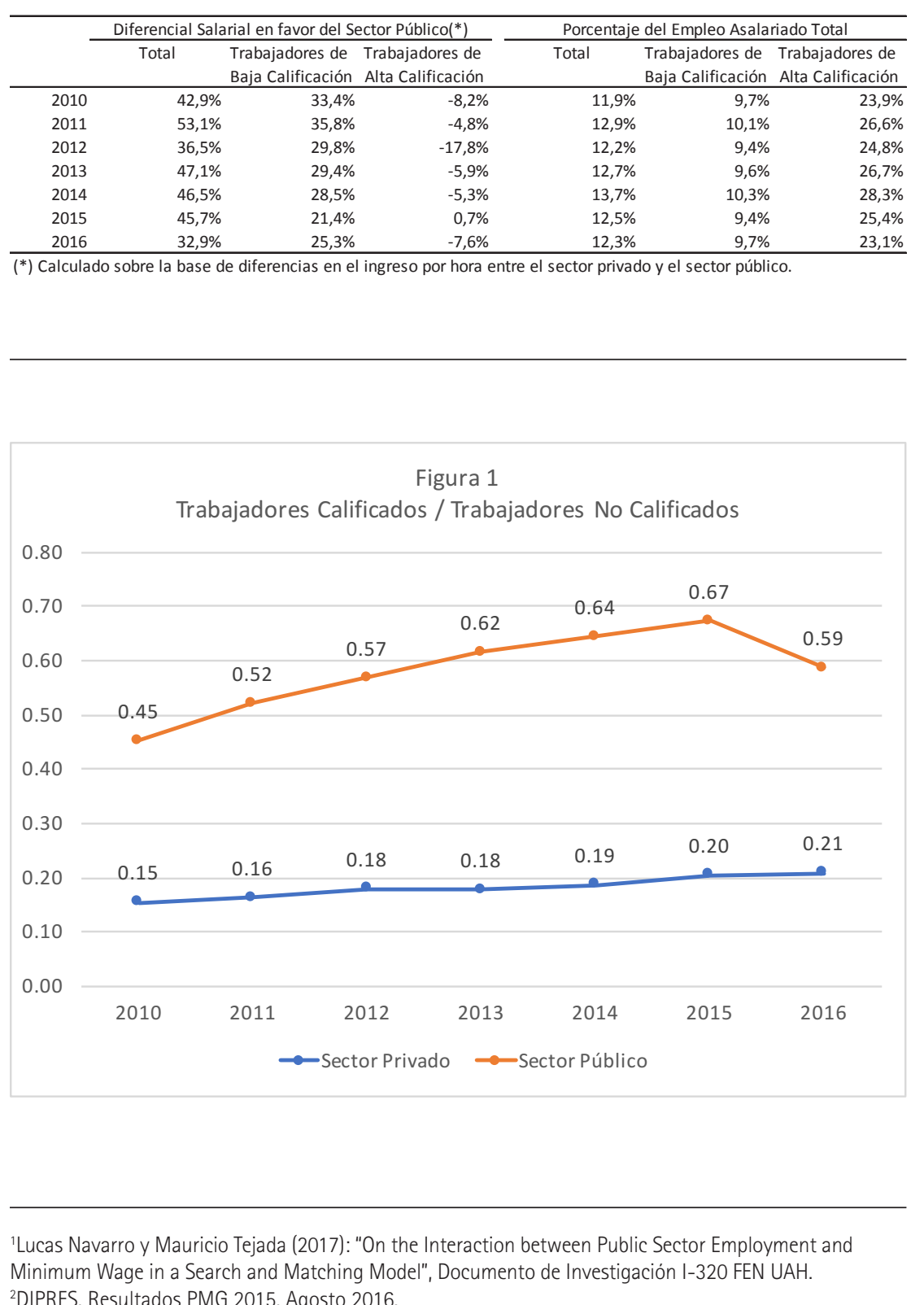

\section{CUÁNTO CRECERÁ CHILE EL 2018}

Por Carlos García, académico FEN UAH.

Una pregunta clave en la actual coyun- de 3,9\%, cifra suficiente para impulsar la fura es si el crecimiento de la economia el demanda interna. Sin que existan nuevas decisiones politicas que los chilenos to- gasto fiscal seria coherente con asegurar mamos eligiendo a Sebastián Piñera como un crecimiento del 30\% en 2018. Presidente por el periodo 2018-2022. Al res- Siguiendo por Lucas, el panorama es pecto, el Banco Central de Chile proyectó a menos claro. Según este economista la principio de diciembre una recuperación el inversión depende de las expectativas de 2018 con un rango para el crecimiento del los empresarios. Por otra parte, los conPIB de 2,5-3,5\%, sin importar la decisión sumidores tambièn definirán su consumo politica, lo que seria sin duda, un salto po- en base a ingreso esperado o riqueza. Si sitivo si se considera el magro crecimiento ambos consideran que las expectativas de 1,5\% promedio de los uiltimos dos años. económicas el 2018 son aún mejores, el La senda de convergencia de la econo- crecimiento del proximo año estaria submía chilena, desde el crecimiento de un va $1,5 \%$ al $3 \%$, depende entre otros factores

En la misma linea de Lucas, un ejemplo neresante de nuestra historia económica erna, básicamente el consumo privado y la fue lo ocurrido con la crisis financiera inSe espera que el consumo privado siga su epicentro en las economias desarrollacreciendo a una tasa del 3\%, por tanto, es das. El mismo Banco Central de Chile proel crecimiento de la inversión sería el que yectaba un crecimiento entre 2-3\% para el marcaría la diferencia. En estos dos últimos 2009, por el contrario, tuvimos una receaños el crecimiento de la inversión fue de sión. En parte, la falla de las proyecciones $-0,8$ y $-2,5 \%$, en cambio ahora se espera del Banco Central fue porque no se tomaque crezca en torno a un $3 \%$.

ron seriamente las recomendaciones de Lucas. Los inversionistas derrumbaron sus versión crezca un 3\% el 2018 sin importar expectativas a causa de la recesion interquien va a gobernar Chile? Incluso, ¿está nacional, y con ello la inversión cayo en un asegurado que el consumo privado sera del estrepitoso $15 \%$. Si bien el consumo siguió $3 \%$ ? La respuesta se puede hacer en base creciendo, el aumento fue modesto $(1,8 \%)$. a los argumentos de los dos más grandes El triunfo de Sebastian Pinera trajo fesión: John Mas que ha tenido la pro- importantes consecuencias para la Bolsa Keys. Ambos ubicados en los extremos, de un $7 \%$ en pocas horas, acenzando su Keynes intervencionista y lucas defensor máximo histórico. Su impacto en la ecoacérrimo del mercado. Partamos por Keynes, en efecto, una po- Lucas: las expectativas aumentan y con lítica expansiva en una situación de bajo ello impulsan no solo al precio de las acEl gobierno anunció en su Ley de Presu- el 2018. puesto de 2018 un crecimiento del gas cercano a los inversionistas tanto nacioextranieros y es muy probale que su gobierno sea muy efectivo en convencerlos a concretar sus proyectos. Con esto, es probable que asegure un crecimiento mayor al 3,5\%, que es el límite Central de Chile.

Por otra parte, el presidente electo tiene todos los incentivos para promover la nversión privada y el empleo. Ėl necesita que la economia chilena crezca para finanlias sus proyectos, es deciri, para recaudor los tribus susicntes que asegure n salud, previsión, etc. Es de esperar, que buen crecimiento del 2018 se concrete reformas sociales y gubernamentales propiadas que mejoren el bienestar de los

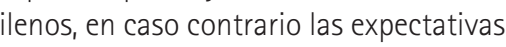
dejarán de ser positivas retornando la tasa

Todo este analisis dentro del supuesto que la economia internacional siga creciendo moderadamente y con ello el precon del cobre se mantenga estable. En caso contrario, todo este artículo serán palabras que se las llevará el viento y no se concremor el an ado el factor intes tendemos a dejar de onseña, que es no solo es importante, s vital.
Decano: Eduardo Abarzúa 